IMMUNE TOLERANCE

\title{
Suicide is painless
}

ce

another

peripheral

tolerance

mechanism

that occurs in

the liver: naive

T cells commit 'suicide' at this

site
A number of peripheral tolerance mechanisms have evolved to silence $\mathrm{T}$ cells that recognize self antigens or other non-infectious antigens that are found outside the thymus, such as food proteins and commensal organisms. Now, researchers have described another peripheral tolerance mechanism that occurs in the liver: naive T cells commit 'suicide' at this site by actively invading antigen-expressing hepatocytes.

The authors transferred naive

DDES T cells (which express a transgenic TCR specific for a self-peptide presented on $\mathrm{H}-2 \mathrm{~K}^{\mathrm{b}}$ ) into $\mathrm{C} 57 \mathrm{BL} / 6$ mice, which ubiquitously express both the self-peptide and $\mathrm{H}-2 \mathrm{~K}^{\mathrm{b}}$. They were surprised to see that these self-reactive $\mathrm{CD} 8^{+} \mathrm{T}$ cells did not promote autoimmune disease. Exploring the fate of the transferred cells, they found that very few DES $\mathrm{T}$ cells could be recovered from the lymph nodes and spleen of the recipient mice, but large numbers were detected in the liver at 1 hour

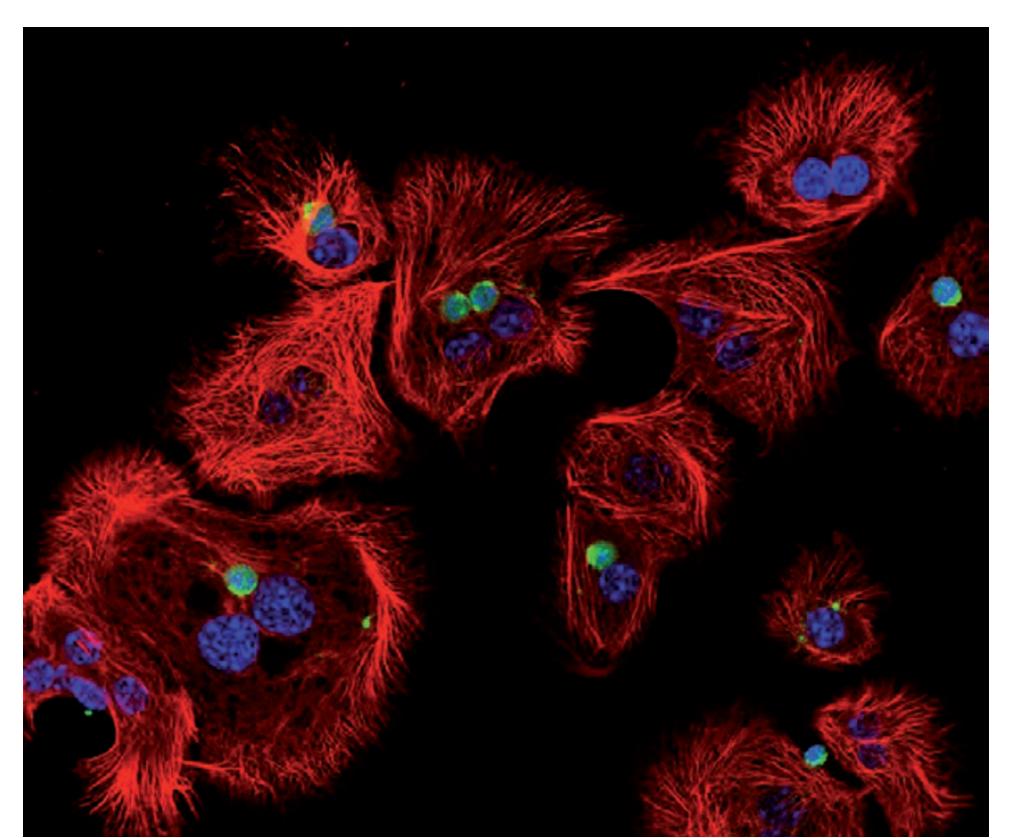

In the image shown, 'suicidal' T cells (green) have invaded co-cultured hepatocytes, which are labelled in red using an antibody specific for cytokeratin 5 and cytokeratin 8 . Cell nuclei are stained in blue using

DAPI. The image appears courtesy of M. Vo and P. Bertolino, University of Sydney, Newtown, Australia.

following transfer. By contrast, in syngeneic B10.BR mice (which do not express $\mathrm{H}-2 \mathrm{~K}^{\mathrm{b}}$ ), few transferred DES T cells were recovered from the liver and instead appeared to home to the lymph nodes and spleen.

Despite the early accumulation of transferred DES T cells in the liver of C57BL/6 recipients, the authors did not detect any sign of liver inflammation. They found that the transferred $\mathrm{T}$ cells were rapidly lost from the liver and that this loss was independent of macrophages and lymphocytes. Studies using annexin-V staining or BCL-2-interacting mediator of cell death (BIM)-deficient DES T cells also indicated that the transferred $\mathrm{T}$ cells were not undergoing apoptosis in the liver. Furthermore, experiments in which DES T cells were labelled with a radioactive isotope before being transferred suggested that $\mathrm{T}$ cell loss from the liver was not due to the redistribution of $\mathrm{T}$ cells to other sites in the body.
So what was happening to the transferred self-reactive T cells? Incredibly, microscopy studies showed DES T cells entering or contained within hepatocytes in the livers of recipient C57BL/6 mice. $\mathrm{T}$ cell remnants could be detected in lysosome-associated membrane glycoprotein 1 (LAMP1) vesicles, suggesting that the $\mathrm{T}$ cells were rapidly destroyed in lysosomes following hepatocyte invasion. In vitro imaging studies revealed that hepatocyte invasion required antigen-specific $\mathrm{T}$ cell activation, as naive DES T cells could be seen entering hepatocytes from $\mathrm{C} 57 \mathrm{BL} / 6$ mice, but not hepatocytes from B10.BR mice. In addition, fixed or heat-inactivated $\mathrm{T}$ cells were not internalized by hepatocytes, suggesting that $\mathrm{T}$ cells were actively invading, rather than being passively engulfed. This conclusion was further supported by the observation that various inhibitors of $\mathrm{T}$ cell activation prevented $\mathrm{T}$ cell invasion. Interestingly, wortmannin, an inhibitor of phosphoinositide 3-kinase, also inhibited T cell entry in hepatocytes in vitro. Finally, the authors showed that this apparent suicide of self-reactive $\mathrm{T}$ cells may contribute to immune tolerance, as treatment of recipient mice with wortmannin led to increased accumulation of donor DES $\mathrm{T}$ cells and the development of an immune-mediated hepatitis.

The term 'emperipolesis' has previously been used to describe how one cell can enter another, but the physiological relevance of this phenomenon has not been clear. The authors propose that the 'suicidal emperipolesis' process they have described may help to prevent immunopathological responses by deleting T cells with specificity for self-proteins or other non-infectious antigens that are not found in the thymus.

Yvonne Bordon

ORIGINAL RESEARCH PAPER Benseler, V. et al. Hepatocyte entry leads to degradation of autoreactive CD8 T cells. Proc. Nat Acad. Sci. USA 108, 16735-16740 (2011) 